\title{
Design of a Rotary Electromagnetic Actuator with Linear Torque Output for Fast Steering Mirror
}

\author{
Yongjun Long ${ }^{1}$, Jinqiu Mo ${ }^{1}$, Xinshu Chen ${ }^{2}$, Qinghua Liang ${ }^{1}$, Yaguang Shang ${ }^{1}$, and Shigang Wang ${ }^{1 *}$ \\ ${ }^{1}$ The State Key Laboratory of Mechanical System and Vibration, School of Mechanical Engineering, Shanghai Jiao Tong University, \\ 800 Dongchuan Road, Shanghai 200240, China \\ ${ }^{2}$ Qiushi College, Tianjin University, 92 Weijin Road, Tianjin 300072, China
}

(Received 5 December 2014, Received in final form 7 March 2015, Accepted 10 March 2015)

\begin{abstract}
This paper focuses on the design of a flux-biased rotary electromagnetic actuator with compact structure for fast steering mirror (FSM). The actuator has high force density and its torque output shows linear dependence on both excitation current and rotation angle. Benefiting from a new electromagnetic topology, no additional axial force is generated and an armature with small moment of inertia is achieved. To improve modeling accuracy, the actuator is modeled with flux leakage taken into account. In order to achieve an FSM with good performance, a design methodology is presented. The methodology aims to achieve a balance between torque output, torque density and required coil magnetomotive force. By using the design methodology, the actuator which will be used to drive our FSM is achieved. The finite element simulation results validate the design results, along with the concept design, magnetic analysis and torque output model.
\end{abstract}

Keywords: fast steering mirror, flux-biased electromagnetic actuator, linear torque output, symmetric electromagnetic structure, optimal design.

\section{Introduction}

As a key component of adaptive optical system, fast steering mirror (FSM) plays a significant role in pointing a beam to a desired place with high accuracy and high response speed and rejecting potential disturbance to achieve very small beam tilting error. The performances of an FSM, such as bandwidth and optical scanning range, are influenced by its actuator greatly. Mostly, FSMs are driven by voice coil actuators [1-4] or piezoelectric actuators $[5,6]$. Voice coil actuator utilizes Lorentz force to generate actuation and allows long actuation stroke with low input power requirement. However, its maximum force output is limited because of the coil overheat problem. Conversely, piezoelectric actuator offers high acceleration and quick response. However, its actuation stroke is short due to its inherent numerous electrical and mechanical losses when operating in high frequency. Therefore, the FSMs using voice coil actuators achieve wider scanning areas but lower bandwidths whereas the

CThe Korean Magnetics Society. All rights reserved.

*Corresponding author: Tel: +86-21-34204496

Fax: +86-21-34204496, e-mail: wangshigang@sjtu.edu.cn piezoelectrically driven FSMs allow higher bandwidths but narrower scanning areas. The ideal alternative to FSM actuator should combine the advantages of both voice coil actuator and piezoelectric actuator.

In another paper of ours [7], we designed a two-axis rotary electromagnetic actuator for our FSM by using the magnetic-flux-biased method which has been successfully used in in fast tool servos [8-10] and nanopositioner [11]. By introducing bias flux and designing proper electromagnetic configuration, the actuator has high force density similar to a solenoid, but its torque output is nearly a linear function of both its driving current and rotation angle. Besides, the effective stroke of the actuator is between the strokes of a piezoelectric actuator and a voice coil actuator. This combination of torque output and stroke indicates that the actuator is ideal for FSM. In fact, two FSMs [12] using flux-biased electromagnetic actuators have been achieved earlier than our FSM. However, as we showed in Ref. [7], the actuators in the two FSMs named the AFSM and SAFSM have some drawbacks such as additional axial force problem, actuator model with low accuracy and so on. By introducing a new electromagnetic topology and taking flux leakage into consideration, an actuator that overcomes the drawbacks 
of the AFSM and SAFSM actuators was achieved in Ref. [7]. In addition, detailed design of the AFSM and SAFSM actuators was not included in Ref. [12].

In Ref. [7], we only focused on the concept design and accurately modeling of our FSM actuator, not including detailed design of the actuator. This paper is motivated by the detailed design of our FSM actuator based on previous study results of Ref. [7]. The designed actuator in this paper aims to accomplish a balance between torque output, required coil magnetomotive force (MMF) and torque density. As a result, an FSM with good performances can be achieved by using the actuator.

\section{Actuator Structure and Model}

First, the basic layout and concept design of our FSM actuator are presented in this section. By introducing a new electromagnetic topology, the actuator overcomes the drawbacks of the AFSM and SAFSM actuators. The actuator is then modeled through the commonly used equivalent magnetic circuit method. As both the permanent magnet (PM) flux and coil flux leakages are modeled properly, an actuator model with high accuracy is obtained. In fact, all the contents in this section have been included in Ref. [7]. Hence, this section presents the analysis in a concise way. More details of the analysis are introduced in Ref. [7].

\subsection{Actuator Concept Design}

Figure 1 shows the structure of our FSM. The movable parts including the mirror, mirror holder and armature are suspended by the flexure support system consisting of the flexible diaphragms and axial flexure. Driven by the electromagnetic actuator consisting of four identical electromagnetic units and an armature, the FSM can generate two rotary motions, $\theta_{X}$ and $\theta_{Y}$, simultaneously. The sensors are used to detect the steering angle in real time to yield closed-loop control.

Figure 2 shows the schematic diagram of the actuator used in the FSM. The actuator overcomes the drawbacks of the AFSM and SAFSM actuators. Benefiting from the "top-bottom" arrangement of the PMs, the actuator achieves a symmetric electromagnetic structure and hence generates no additional axial force. Moreover, a high bias flux density can be achieved in each air gap without requiring big PMs due to the "top-bottom" arrangement. The cores and armature are all made of materials with high magnetic permeability to improve electromagnetic efficiency. Unlike the ring armature used in the SAFSM [12], the actuator is with a new cross topology armature. In Ref. [7], we have proved that the cross topology armature has smaller moment of inertia than the ring topology armature used in the SAFSM if they can conduct the same maximum amount of magnetic flux. As a result, a higher acceleration and bandwidth of our FSM can be achieved.

Figure 3 shows the coil and PM fluxes related to the steering motion $\theta_{X}$. The PMs generate DC bias flux in each air gap and the coil windings produce time-varying coil flux. Magnetic flux in each air gap is the super-

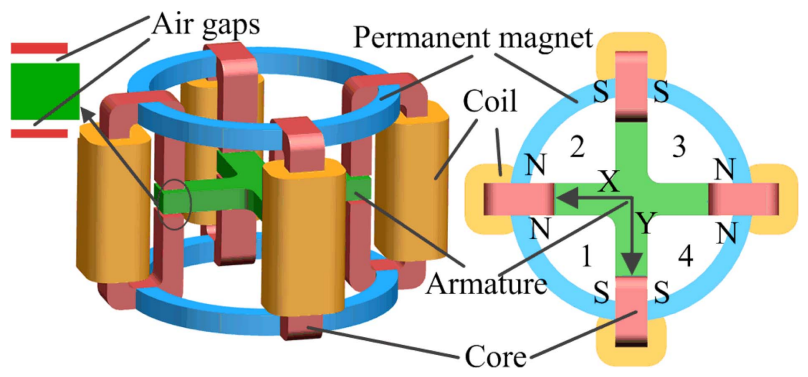

Fig. 2. (Color online) Schematic diagram of the actuator.

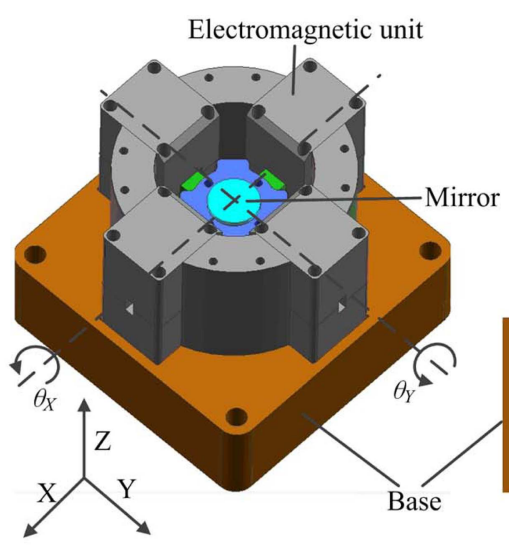

(a)

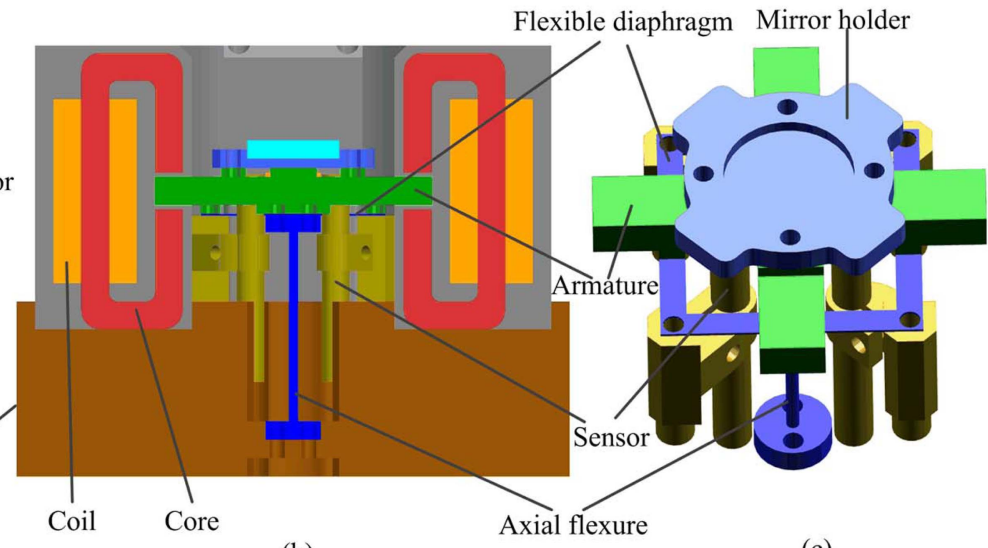

(b) (c)

Fig. 1. (Color online) Structure overview of our FSM. (a) Three-dimensional CAD shape of the FSM, (b) cross-sectional view of the FSM, (c) flexure support system of the FSM. 


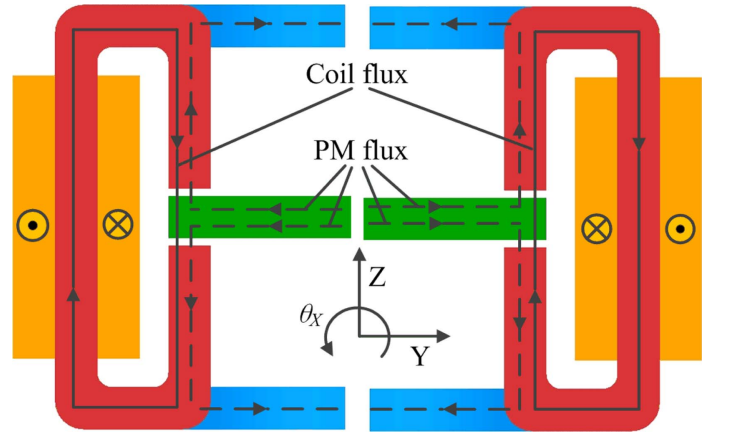

Fig. 3. (Color online) Coil and PM fluxes related to the steering motion $\theta_{X}$.

position of coil flux and PM bias flux. Assigning two currents in the directions shown in Fig. 3 to the two coils causes the increase of the fluxes in the right upper and left lower air gaps and the decrease of the fluxes in the right lower and left upper air gaps at the same time. Hence, the two left air gaps generate a force pointing to the negative Z-direction while the two right air gaps generate a force pointing to the positive Z-direction. Consequently, a torque pointing to the positive X-direction is generated on the armature and a positive steering angle $\theta_{X}$ is then produced. Because of the symmetry of the actuator, the operating principle of the steering motion $\theta_{Y}$ is the same as that of the steering motion $\theta_{X}$.

\subsection{Actuator Model}

The ideal equivalent magnetic circuit model of the actuator is shown by Fig. 4. Flux leakage, fringing loss and the reluctances of the cores and armature are ignored in the ideal model. Each PM is modeled as a MMF source with internal reluctance. For example, $R_{1 U}$ and $\Psi_{1 U}$ respectively denote the reluctance and MMF of the upper PM in the area of "1" (refer to Fig. 2), $R_{1 L}$ and $\Psi_{1 L}$ respectively denote the reluctance and MMF of the lower PM in the area of "1", and so on. Each excitation coil is modeled as a coil MMF. For example, $\Psi_{+Y}$ indicates the MMF of the coil in the positive Y-direction, $\Psi_{-Y}$ indicates the MMF of the coil in the negative Y-direction, and so on. In our design, the two coils that control the same steering motion are physically wired together, trying to eliminate additional force. Therefore, $\Psi_{+Y}$ is equal to $\Psi_{-Y}$ and $\Psi_{+X}$ is equal to $\Psi_{-X}$. Each air gap is modeled as a reluctance. For example, $R_{+Y U}$ is the reluctance of the upper air gap in the positive Y-direction, $R_{+Y L}$ is reluctance of the lower air gap in the positive Y-direction, and so on.

Solving the magnetic circuit gives the ideal bias flux density and ideal coil flux density in each air gap. Details of solving the magnetic circuit are provided in Ref. [7].

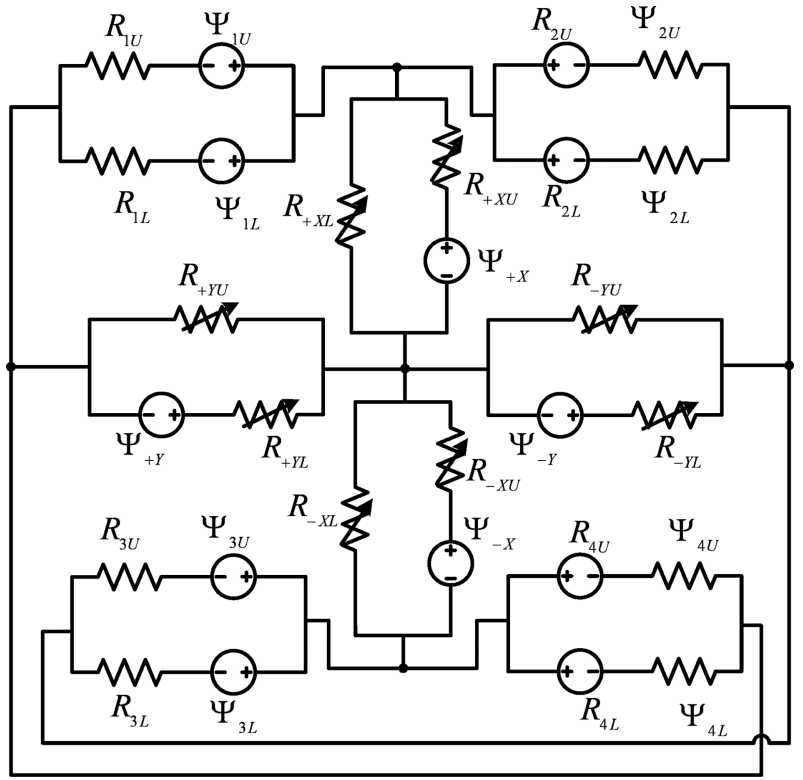

Fig. 4. Ideal equivalent magnetic circuit of the actuator.

Because of the symmetry of the actuator, only the steering motion $\theta_{X}$ is introduced in the following analysis.

Solving the magnetic circuit, we can derive the ideal bias flux density and ideal coil flux density of each air gap as:

$$
\begin{aligned}
& B_{-Y L}^{P M}=B_{+Y U}^{P M}=\frac{L_{0}+\theta_{X} r}{L_{0}} \cdot B_{P M} \\
& B_{-Y L}^{P M}=B_{+Y L}^{P M}=\frac{L_{0}+\theta_{X} r}{L_{0}} \cdot B_{P M} \\
& B_{P M}=\frac{2 B_{r} A_{P M}}{A_{g}} \\
& B_{+Y U}^{\text {Coil }}=B_{+Y L}^{\text {Coil }}=B_{-Y U}^{\text {Coil }}=B_{-Y L}^{\text {Coil }}=\frac{N \mu_{0}}{2 L_{0}} I_{Y}
\end{aligned}
$$

Here $B_{-Y L}^{P M}$ is the ideal bias flux density of the " $-Y$ " lower air gap, $B_{+Y U}^{P M}$ is the ideal bias flux density of the " $+Y$ " upper air gap and so on. $B_{-Y L}^{\text {Coil }}$ is the ideal coil flux density of the " $-Y$ " lower air gap, $B_{-Y U}^{\text {Coil }}$ is the ideal coil flux density of the "+Y" upper air gap and so on. $A_{P M}$ and $B_{r}$ are the identical pole area and remanence of the PMs respectively, $L_{0}$ is the initial air gap length, $A_{\mathrm{g}}$ is the air gap pole area, $r$ is the effective radius of rotation, $N$ is the number of coil turns, $I_{Y}$ is the identical driving current in the " $+Y$ " and " $-Y$ " coils, $\mu_{0}$ is the permeability of vacuum.

The ideal flux density is derived without taking flux leakage into consideration. However, the actuator's torque output is greatly affected by flux leakage, especially the PM flux leakage. To improve modeling accuracy, both the PM flux and coil flux leakages are modeled properly 


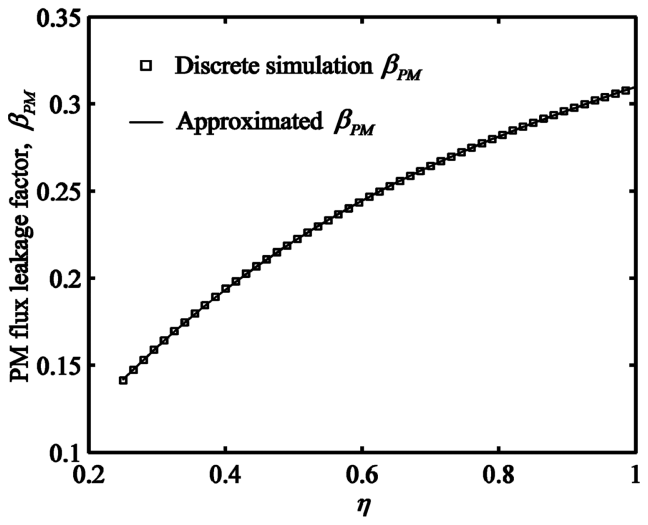

(a)

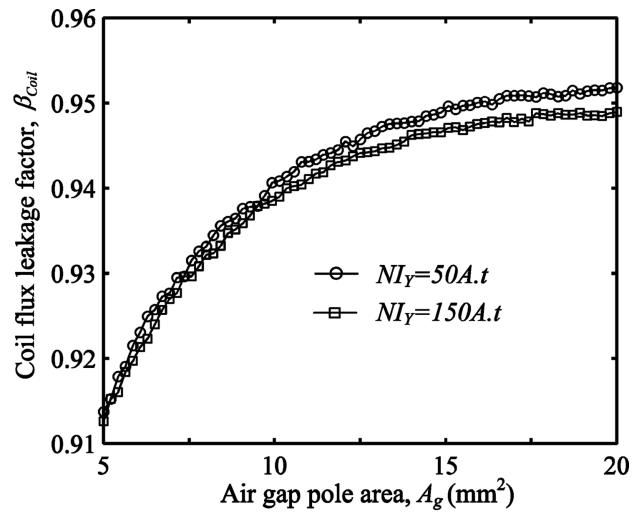

(b)

Fig. 5. PM flux and coil flux leakage factors. (a) Approximated PM flux leakage factor, (b) simulation coil flux leakage factor.

through finite element simulations. Details of modeling the PM flux and coil flux leakages are provided in Ref. [7].

The PM flux leakage factor $\beta_{P M}$ defined as the ratio of real PM flux to ideal PM flux is fitted by using a cubic polynomial function. The approximated $\beta_{P M}$ is

$$
\begin{aligned}
\beta_{P M}(\eta)= & 0.13062 \eta^{3}-0.41613 \eta^{2}+0.57295 \eta \\
& +0.02241
\end{aligned}
$$

where $\eta=A_{g} / A_{\max }$ and $A_{\max }=20 \mathrm{~mm}^{2}$. Fig. 5(a) plots the curve of $\beta_{P M}(\eta)$ along with the discrete simulation $\beta_{P M}$, indicating the effectiveness of the curve fitting results.

The coil flux leakage factor $\beta_{\text {Coil }}$ defined as the ratio of real coil flux to ideal coil flux varies slightly. Fig. 5(b) shows the simulation results of $\beta_{\text {Coil }}$.

The real total flux density in each air gap is the superposition of the real PM flux density and real coil flux density. Using the PM flux leakage factor $\beta_{P M}$ and coil flux leakage factor $\beta_{\text {Coil }}$ to modify the ideal flux density, we derive the real total flux densities in the " $+Y$ " and " $-Y$ " air gaps as:

$$
\begin{aligned}
B_{+Y U} & =B_{-Y L}=\beta_{P M} B_{+Y U}^{P M}+\beta_{C o i l} B_{+Y U}^{C o i l} \\
& =\beta_{P M} B_{P M} \cdot \frac{L_{0}+\theta_{X} r}{L_{0}}+\frac{\beta_{C o i l} N \mu_{0}}{2 L_{0}} I_{Y} \\
B_{+Y L} & =B_{-Y U}=\beta_{P M} B_{+Y L}^{P M}-\beta_{C o i l} B_{+Y L}^{C o i l} \\
& =\beta_{P M} B_{P M} \cdot \frac{L_{0}+\theta_{X} r}{L_{0}}+\frac{\beta_{C o i l} N \mu_{0}}{2 L_{0}} I_{Y}
\end{aligned}
$$

where $B_{+Y U}$ is the real total flux density in the " $+Y$ " upper air gap, $B_{-Y L}$ is the real total flux density in the " $-Y$ " lower air gap, and so on.

Hence, we can derive the electromagnetic forces generated by the two " $Y$ " air gaps and two " $-Y$ " air gaps respectively

$$
\begin{aligned}
F_{+Y}= & F_{-Y}=A_{g} \frac{\left(B_{+Y U}\right)^{2}-\left(B_{+Y L}\right)^{2}}{2 \mu_{0}}=\frac{2 \beta_{P M}^{2} A_{g} B_{P M}^{2} r}{L_{0} \mu_{0}} \theta_{X} \\
& +\frac{N \beta_{P M} \beta_{C o i l} B_{P M} A_{g}}{L_{0}} I_{Y}
\end{aligned}
$$

$F_{+Y}$ and $F_{-Y}$ are equal and opposite. Hence, the net force acting on the armature is null and a torque pointing to positive X-direction is generated on the armature. Multiplying $F_{+Y}$ by $2 r$ gives the actuating torque output

$$
T_{X}=2 F_{+Y} r=K_{\theta} \theta_{X}+K_{I} I_{Y}
$$

where

$$
\begin{aligned}
K_{\theta} & =\frac{4 A_{g} \beta_{P M}^{2} B_{P M}^{2} r^{2}}{L_{0} \mu_{0}} \\
K_{I} & =\frac{2 \beta_{P M} \beta_{C o i l} A_{g} B_{P M} N r}{L_{0}}
\end{aligned}
$$

$K_{\theta}$ and $K_{I}$ are constants, indicating that the torque output of the actuator is a linear function of both its driving current and rotation angle.

\section{Actuator Design}

Detailed design of the AFSM and SAFSM actuators was not included in Ref. [12]. To achieve an FSM with good performances, optimal design of FSM actuator is required. In this paper, the designed actuator aims to accomplish a balance between torque output, required coil MMF and torque density.

\subsection{Design Goals}

To achieve high dynamic stiffness of the actuator, a maximum torque output is preferable. Besides, a larger 
torque output allows a flexure support system with higher stiffness. As a result, a flexure support system with higher strength can be achieved, therefore improving the fatigue life of the flexure support system.

The maximum achievable torque output of a designed actuator is achieved when the flux density in one air gap reaches the saturation flux density of the core material or when the flux density in one air gap becomes zero. That is, the maximum achievable torque output is achieved when

$$
\left\{\begin{array}{l}
B_{+Y U}=\beta_{P M} B_{P M} \cdot \frac{L_{0}+\theta_{X} r}{L_{0}}+\frac{\beta_{C o i l} N \mu_{0}}{2 L_{0}} I_{Y}=B_{S} \\
0 \leq B_{+Y L}=\beta_{P M} B_{P M} \cdot \frac{L_{0}-\theta_{X} r}{L_{0}}-\frac{\beta_{C o i l} N \mu_{0}}{2 L_{0}} I_{Y}<B_{S}
\end{array}\right.
$$

or when

$$
\left\{\begin{array}{l}
0<B_{+Y U}=\beta_{P M} B_{P M} \cdot \frac{L_{0}+\theta_{X} r}{L_{0}}+\frac{\beta_{C o i l} N \mu_{0}}{2 L_{0}} I_{Y} \leq B_{S} \\
B_{+Y L}=\beta_{P M} B_{P M} \cdot \frac{L_{0}-\theta_{X} r}{L_{0}}-\frac{\beta_{C o i l} N \mu_{0}}{2 L_{0}} I_{Y}=0
\end{array}\right.
$$

where $B_{s}$ is the saturation flux density of the core and armature material.

Substituting Eq. (12) and Eq. (13) into Eq. (8) and Eq. (9) gives the maximum achievable torque output

$$
T_{\max }=\left\{\begin{array}{l}
\frac{B_{s}^{2}-\left(2 \beta_{P M} B_{P M}-B_{S}\right)^{2}}{\mu_{0}} r A_{g}\left(B_{S} \leq 2 \beta_{P M} B_{P M}<2 B_{S}\right) \\
\frac{\left(2 \beta_{P M} B_{P M}\right)^{2}}{\mu_{0}} r A_{g}\left(0<2 \beta_{P M} B_{P M} \leq B_{S}\right)
\end{array}\right.
$$

For convenience of analysis, variable $\gamma$ representing the strength of the bias PM flux is introduced and is defined by

$$
\gamma=\frac{B_{P M}}{\left(\frac{B_{S}}{2 \beta_{P M}}\right)}
$$

Hence, Eq. (14) can be changed to

$$
T_{\max }=\left\{\begin{array}{l}
\frac{r A_{g} B_{s}^{2}}{\mu_{0}}\left(2 \gamma-\gamma^{2}\right)(1 \leq \gamma<2) \\
\frac{r A_{g} B_{s}^{2}}{\mu_{0}} \gamma^{2}(0<\gamma \leq 1)
\end{array}\right.
$$

From Eq. (16), it can be seen that increasing the maximum torque output may cause an armature with large moment of inertia. As a result, the FSM acceleration and bandwidth may decrease. For convenience of analysis, the torque density which is similar to the force density in Ref. [9] is defined as

$$
D_{T}=\frac{T_{X}}{J}
$$

where $J$ is the moment of inertia of the armature. Obviously, a larger $D_{T}$ causes a higher acceleration and smaller armature size. Hence, a maximum torque density is preferable from the perspective of dynamic performance.

Designing the cross armature with square section, we derive the moment of inertia of the actuator as

$$
J=\frac{\rho d A_{a}\left(d^{2}+3 A_{a}\right)}{12}-\frac{\rho A_{a}^{2} \sqrt{A_{a}}}{6} \approx \frac{\rho d A_{a}\left(d^{2}+3 A_{a}\right)}{12}
$$

where $A_{a}$ is the armature cross-sectional area, $d$ is the armature length and $\rho$ is the mass density of the armature material.

The armature cross-sectional area $A_{a}$ should be designed large enough to conduct the bias PM flux and to prevent flux saturation in the armature. Hence, the following is obtained

$$
A_{a} B_{S} \geq \beta_{P M} A_{g}\left(B_{+Y U}^{P M}+B_{+Y L}^{P M}\right)
$$

Combing Eqs. (1)-(3), Eq. (15), and Eq. (19), we can derive that

$$
A_{a} \geq \gamma A_{g}
$$

Here, we design $A_{a}=1.1 \gamma A_{g}$.

Substituting $A_{a}=1.1 \gamma A_{g}$ into Eq. (18) and combing Eqs. (9)-(11), Eq. (15), and Eqs. (17)-(18), we change the representation of the torque density to

$$
\begin{aligned}
D_{T}= & \frac{12 B_{S}^{2} r^{2} \theta_{X}}{11 L_{0} \mu_{0} \rho d} \cdot \frac{\gamma^{2}}{\left(d^{2}+3.3 \gamma A_{g}\right)} \\
& +\frac{12 B_{S} \beta_{\text {Coil }} N r I_{Y}}{11 L_{0} \rho d} \cdot \frac{\gamma}{\left(d^{2}+3.3 \gamma A_{g}\right)}
\end{aligned}
$$

Increasing the maximum torque and torque density may require a larger coil MMF. Too large coil MMF is not allowed as it is prohibitive for the design of the power amplifier used to drive the actuator. According to Faraday's Law, the terminal voltage across one coil is

$$
V=-\frac{d\left(N \Phi_{c o i l}\right)}{d t}=-\frac{d\left(N_{g} B_{+Y U}^{C o i l}\right)}{d t}
$$

Substituting Eq. (4) into Eq. (22) gives

$$
V=-L \frac{d}{d t}\left(I_{Y}\right)
$$


where

$$
L=\frac{A_{g} N^{2} \mu_{0}}{2 L_{0}}
$$

$L$ is the coil inductance and is proportional to the square of the coil turns. From Eq. (23) and Eq. (24), we know that a powerful amplifier is required to achieve fast current response if too large coil MMF is required. Hence, a minimum coil MMF is preferable from the perspective of designing power amplifier.

The required coil MMF $\Psi_{r}$ can be given according to the torque requirement $T_{r}$ which is determined by the flexure support system. Setting the armature at its center position $\left(\theta_{X}=0\right)$ and combining Eq. (3), Eqs. (9)-(11), and Eq. (15), we can derive the required coil MMF as

$$
\Psi_{r}=\frac{T_{r} L_{0}}{A_{g} \beta_{\text {Coil }} B_{S} \gamma r}
$$

The designed actuator should achieve a balance between torque output, torque density and required coil MMF.

\subsection{Structural Parameters Design}

Based on the previous analysis, design of the actuator's structural parameters is presented. The main design parameters include the initial air gap length $L_{0}$, air gap pole area $A_{g}$ and bias flux strength $\gamma$.

\subsubsection{Initial Air Gap Length}

The minimum initial air gap length should meet the FSM angular range requirement $\theta_{r}$ of $\pm 10 \mathrm{mrad}$. Hence, the designed initial air gap length $L_{0}$ should meet that

$$
L_{0} \geq 0.5 d \theta_{r}
$$

The armature length $d$ is mainly determined by the flexure support system and angle detecting system and has been designed to be $25 \mathrm{~mm}$. Therefore, the initial air gap length should be designed to be larger than $0.125 \mathrm{~mm}$ to avoid interference. However, it can be seen that increasing the initial air gap length leads to the decrease of the torque output, decrease of the torque density and increase of the required coil MMF from Eqs. (9)-(11), Eq. (21) and Eq. (25). Hence, too large initial air gap length is not allowed. For some unpredictable errors and the potential increase of angular range, the initial air gap length is designed to be $0.3 \mathrm{~mm}$.

\subsubsection{Bias Flux Strength}

The bias flux strength $\gamma$ determines the bias PM flux density in each air gap. Taking the partial differentials of Eq. (21) and Eq. (25) with respect to $\gamma$ gives

$$
\left\{\begin{array}{l}
\frac{\partial D_{T}}{\partial \gamma}>0 \\
\frac{\partial \Psi_{r}}{\partial \gamma}<0
\end{array}\right.
$$

From (27), we know that a larger $\gamma$ is preferable from the perspective of increasing torque density and decreasing required coil MMF. However, Fig. 6 shows that $T_{\max }$ has its maximum value $T_{M}$ when setting $\gamma=1$. Increasing the bias flux strength $\gamma$ causes the decrease of the maximum achievable torque output. To make sure that the maximum achievable torque output is no less than 80 percent of $T_{M}$, the design domain of the bias flux strength $\gamma$ is limited by $1 \leq \gamma \leq 1.44$.

From Fig. 6, it can be seen that when $\gamma$ changes from 1 to 1.3 ( $\gamma$ changes 0.3 ), $T_{\max }$ only changes $0.1 T_{\mathrm{M}}$ whereas when $\gamma$ changes from 1.3 to 1.44 ( $\gamma$ only changes 0.14 ), $T_{\max }$ also changes $0.1 T_{\mathrm{M}}$.

Fig. 7 shows the variation of required coil MMF with respect to bias flux strength. From Fig. 7, it can be seen that when $\gamma$ changes from 1 to 1.3 ( $\gamma$ changes 0.3 ), $\Psi_{r}$ has a significant decrease of $0.23 \Psi_{1}$ whereas when $\gamma$ changes

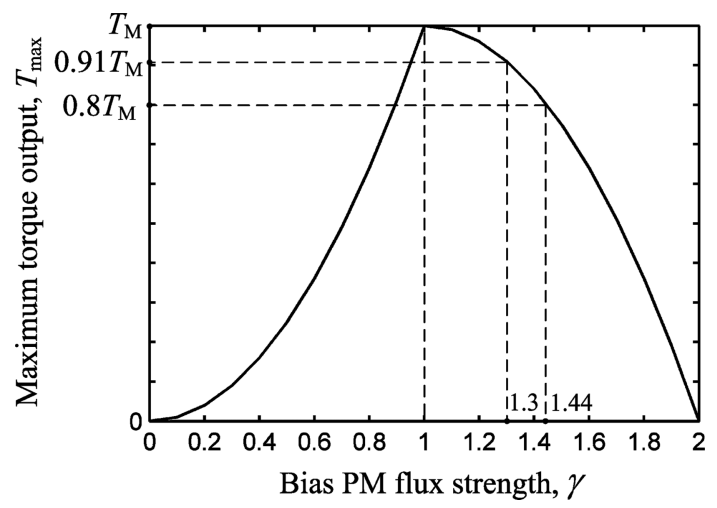

Fig. 6. Variation of maximum achievable torque with bias PM flux strength.

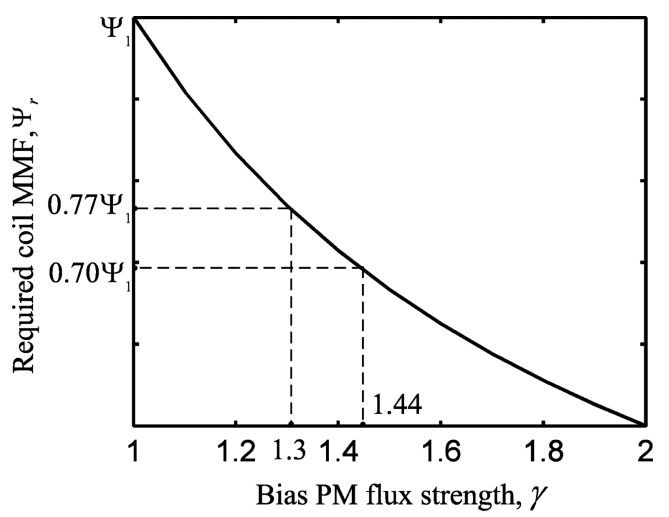

Fig. 7. Variation of required coil MMF with bias PM flux strength. 


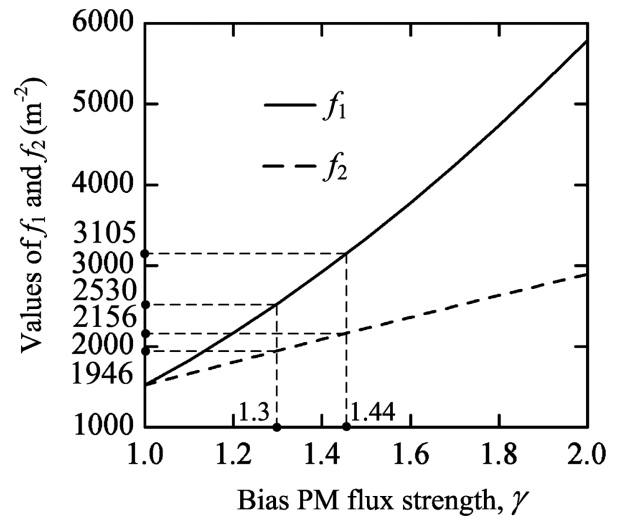

Fig. 8. Variation of $f_{1}$ and $f_{2}$ with bias PM flux strength.

from 1.3 to 1.44 ( $\gamma$ changes 0.14$), \Psi_{r}$ has only a slight decrease of $0.07 \Psi_{1}$.

From Eq. (21), we know that the effect of bias strength $\gamma$ on torque density can be analyzed through studying the variation of the following two functions with respect to $\gamma$.

$$
\begin{aligned}
& f_{1}=\frac{\gamma^{2}}{d^{2}+3.3 \gamma A_{g}} \\
& f_{2}=\frac{\gamma}{d^{2}+3.3 \gamma A_{g}}
\end{aligned}
$$

If setting $A_{g}=10 \mathrm{~mm}^{2}$, we can obtain the curves of $f_{1}$ and $f_{2}$ as Fig. 8 shows. From Fig. 8 , we know that $f_{1}$ and $f_{2}$ change significantly when $\gamma$ changes from 1 to 1.3 whereas $f_{1}$ and $f_{2}$ change slightly when $\gamma$ changes from 1.3 to 1.44 . Designing $A_{g}$ to be other value gives the same analysis result.

Based on the analysis on Figs. 6, Fig. 7 and Fig. 8, it can be concluded that the maximum torque output has a slight change of 10 percent of the maximum achievable torque $T_{M}$ whereas the torque density and required coil MMF change significantly when $\gamma$ changes from 1 to 1.3 . When $\gamma$ changes from 1.3 to 1.44 , the maximum torque output also has a change of $0.1 T_{M}$ whereas the torque density and required coil MMF change slightly. Considering the conclusion, we design $\gamma=1.3$ which guarantees a reasonable balance between torque output, torque density and required coil MMF.

\subsubsection{Air Gap Pole Area}

Limited by fabrication problem and FSM size requirement, design domain of the air gap pole area $A_{g}$ is set to be $5 \mathrm{~mm}^{2}<A_{g}<20 \mathrm{~mm}^{2}$ in which enough torque output is ensured to drive the FSM. The cores and the armature are laminated with nickel-iron alloy whose saturation flux density $B_{s}$ is about $1.4 \mathrm{~T}$. The effective radius $r$ is the distance from the rotation center to the air gap pole face

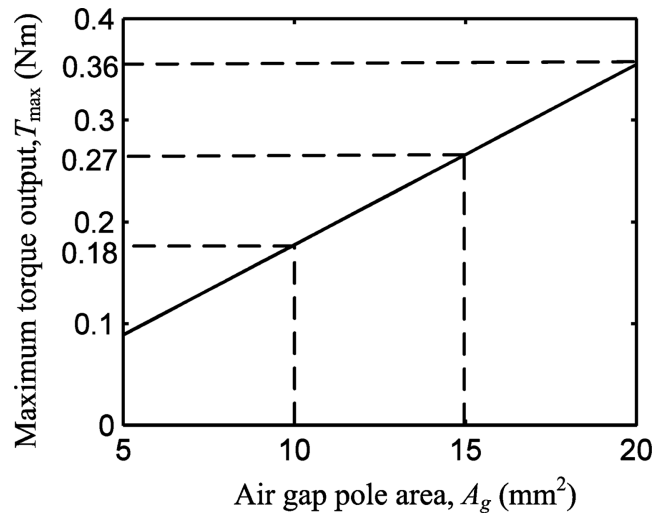

Fig. 9. Variation of maximum achievable torque with air gap pole area.

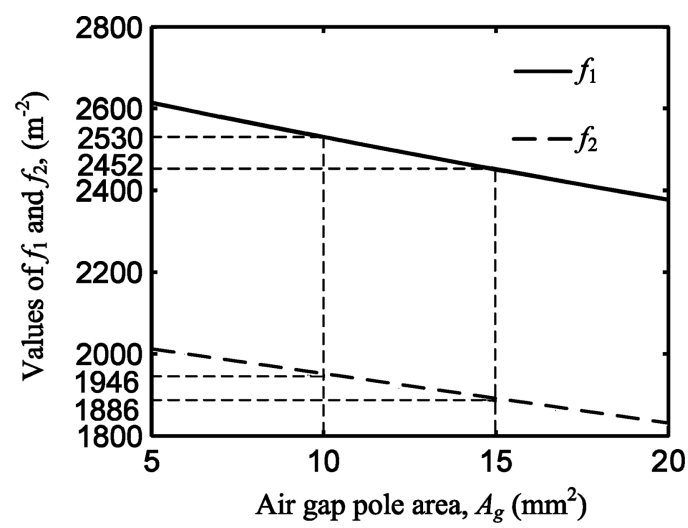

Fig. 10. Variation of $f_{1}$ and $f_{2}$ with air gap pole area.

center. It equals to half of the armature length minus half of the length of the air gap pole face. Before designing $r$ in detail, it can be approximated by $0.5 \mathrm{~d}(12.5 \mathrm{~mm})$ conservatively. Determined by the flexure support system, a minimum torque output $T_{r}$ of 0.05 N.m is required. Referring to Fig. 5(b), the coil leakage factor $\beta_{\text {Coil }}$ is assumed to be 0.94 .

Figs. 9, Fig. 10 and Fig. 11 show the effects of the air

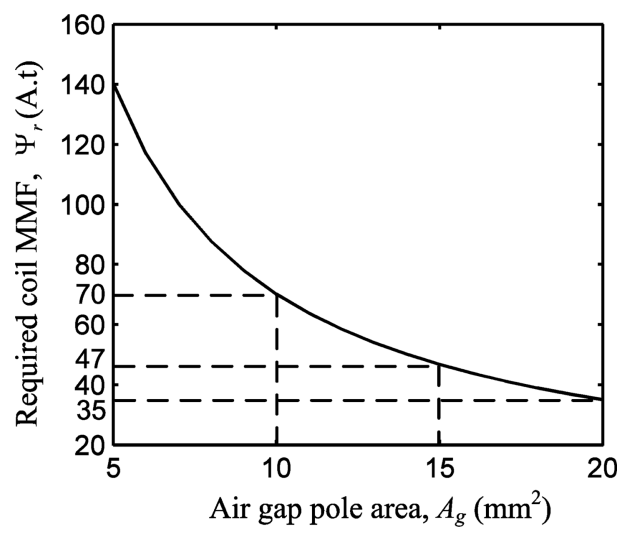

Fig. 11. Variation of required coil MMF with air gap pole area. 
gap pole area $A_{g}$ on $T_{\max }, f_{1}$ and $f_{2}$, and $\Psi_{r}$ respectively. From Fig. 9, it can be seen that $T_{\max }$ is proportional to $A_{g}$. After analyzing Fig. 10, one can easily obtain that $f_{1}$ and $f_{2}$ decrease with $A_{g}$ nearly linearly in the design domain of $A_{g} . \Psi_{r}$ is inversely proportional to $A_{g}$. From Fig. 11, it can be concluded that $\Psi_{r}$ changes significantly in the domain $5 \mathrm{~mm}^{2}<A_{g}<10 \mathrm{~mm}^{2}$ in which the maximum achievable torque output is far larger than the minimum required torque. Considering these analysis, we design the air gap pole area $A_{g}$ to be $9 \mathrm{~mm}^{2}$ to maintain a relatively large torque density. Hence, the maximum achievable torque output and minimum required coil MMF are obtained to be $0.16 \mathrm{~N} . \mathrm{m}$ and 78 A.t, respectively.

\subsubsection{Other Design Parameters}

By combing Eq. (3) and Eq. (15), the PM pole area $A_{P M}$ can be given by

$$
A_{P M}=\frac{\gamma B_{S} A_{g}}{4 \beta_{P M} B_{r}}
$$

The remanence of the PMs (neodymium-iron-boron type) is $1.2 \mathrm{~T}$. Substituting $\eta=0.45\left(A_{g}=9 \mathrm{~mm}^{2}\right)$ into Eq. (5) gives that $\beta_{P M}=0.21$. Hence, the PM pole area $A_{P M}$ is obtain to be $16.3 \mathrm{~mm}^{2}$. The PM length determines the PM MMF. Limited by the FSM structure, the PM length is designed to be $10 \mathrm{~mm}$, which is adequate to provide enough bias MMF, by using finite element simulation. Because of fabrication problem, each curved permanent magnet is replaced by the combination of two cube permanent magnets whose lengths are both $5 \mathrm{~mm}$.

As we design $A_{a}=1.1 \gamma A_{g}$ in Sec. 3.1, the armature cross-sectional area $A_{a}$ is obtained to be $13 \mathrm{~mm}^{2}$. Making a balance between coil turns and excitation current, the coil turns $N$ and minimum required excitation current $I_{Y}$ are obtained to be 26 and 3 A, respectively.

As a summary, Table 1 gives the design results of the FSM actuator.

\section{Validation and Analysis through Finite Element Simulations}

In order to validate the concept design, magnetic analysis,

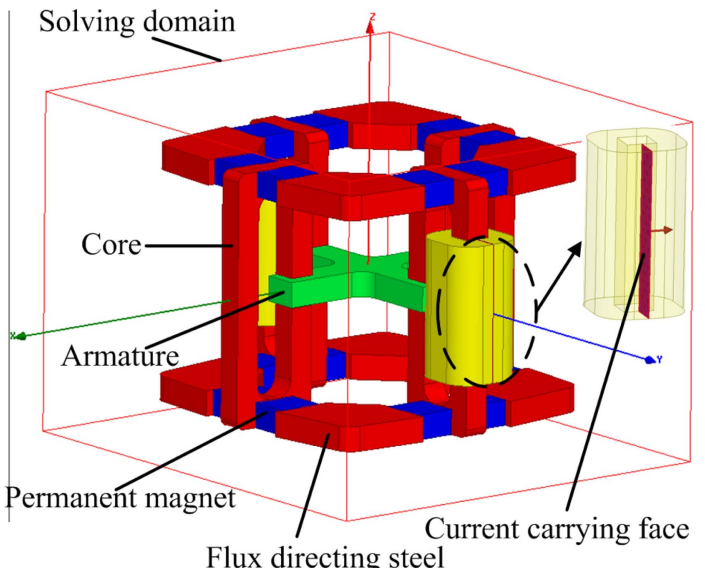

Fig. 12. (Color online) Magnetic finite element simulation model.

torque output model and actuator design results, a 3-D magnetic model for finite element simulations is created based on the designed structural parameters of the FSM actuator. Then a large number of simulations with varying coil MMFs and varying rotation angles are carried out by using the parametric simulation function of Ansoft Maxwell 15.0 to obtain the additional axial forces, and torque outputs.

The 3-D finite element simulation model is shown by Fig. 12. As only the steering motion $\theta_{X}$ is concerned, only the coils in the Y-direction are added to the finite element analysis model to decrease the total 3-D elements. Because of fabrication problem, two cube permanent magnets each of which has a length of $5 \mathrm{~mm}$ are used to replace each curved permanent magnet. A cubic with 'vacuum' material assigned is created to restrict the simulation solving domain. The current carrying face is used to assign current excitation and is obtained by cutting the coil vertically through the $\mathrm{Y}$ axis.

Ansoft Maxwell can mesh automatically, without the need of manually meshing. It will refine the mesh adaptively until the output variable converges sufficiently. However, initial mesh of the finite element simulation model is achieved manually in this paper to realize high computation accuracy. Especially the faces at the ends of the air gaps, a fine mesh is assigned. Figure 13 shows the

Table 1. Design results of the FSM actuator.

\begin{tabular}{cccc}
\hline \hline Parameter & Value & Parameter & Value \\
\hline Armature length, $d$ & $25 \mathrm{~mm}$ & Permanent magnet length, $L_{P M}$ & $10 \mathrm{~mm}$ \\
Initial gap length, $L_{0}$ & $0.3 \mathrm{~mm}$ & Required coil MMF, $\Psi_{r}$ & 78 A.t \\
Bias flux strength, $\gamma$ & 1.3 & Coil turns, $N$ & 26 \\
Air gap pole area, $A_{g}$ & $9 \mathrm{~mm}^{2}$ & Required excitation current, $I_{Y}$ & $3 \mathrm{~A}$ \\
Permanent magnet pole area, $A_{P M}$ & $16.3 \mathrm{~mm}^{2}$ & Armature cross-sectional area, $A_{a}$ & $13 \mathrm{~mm}^{2}$ \\
\hline
\end{tabular}




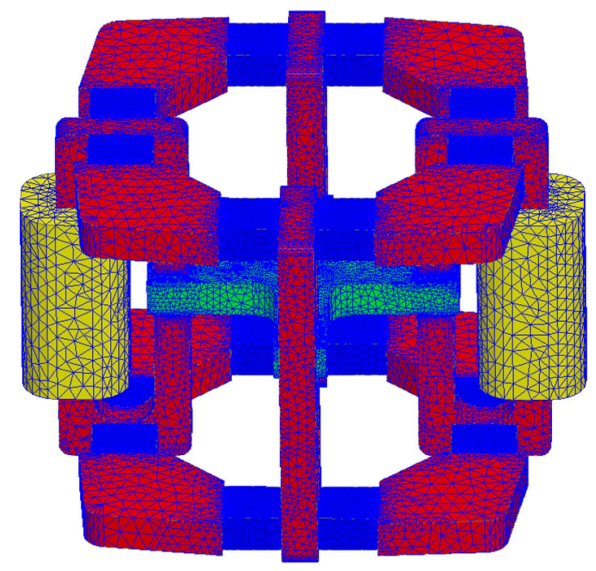

Fig. 13. (Color online) Initial mesh of the finite element simulation model.

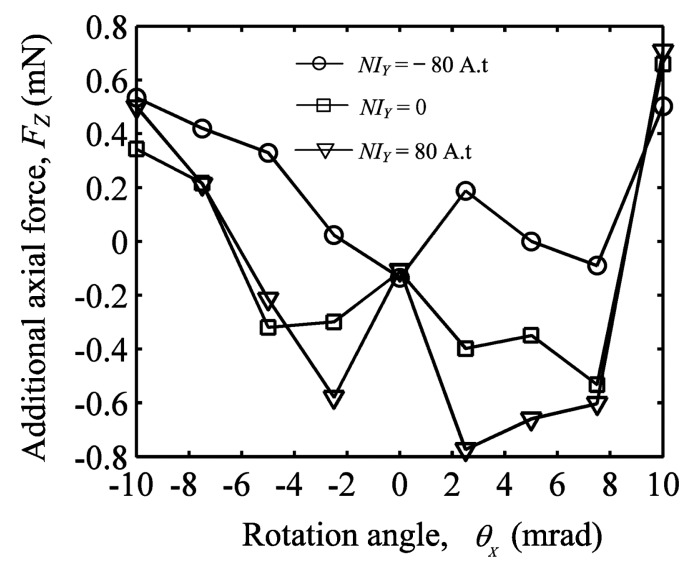

Fig. 14. Additional axial force obtained via simulation.

initial mesh. Besides, the 'percent error' is set to be 0.05 to improve computation accuracy.

The additional axial forces obtained through simulations are shown by Fig. 14. Obviously, the additional axial forces of the designed actuator are very small and are on the order of micronewton. The simulation additional axial forces are caused by simulation errors, not generated by the actuator itself. Smaller additional axial forces will be obtained if a finer initial mesh is assigned to the finite element analysis model and the 'percent error' is set to be a smaller value. However, the computation cost will increase.

The relationship between the coil MMF and torque output, including the simulation torque output and analytical torque output, is shown by Fig. 15. The simulation torque outputs validate the effectiveness and accuracy of the analytical actuator model and confirm the liner relationship between the actuator's torque output and excitation current. As both the PM flux and coil flux leakages are taken into account in magnetic analysis and

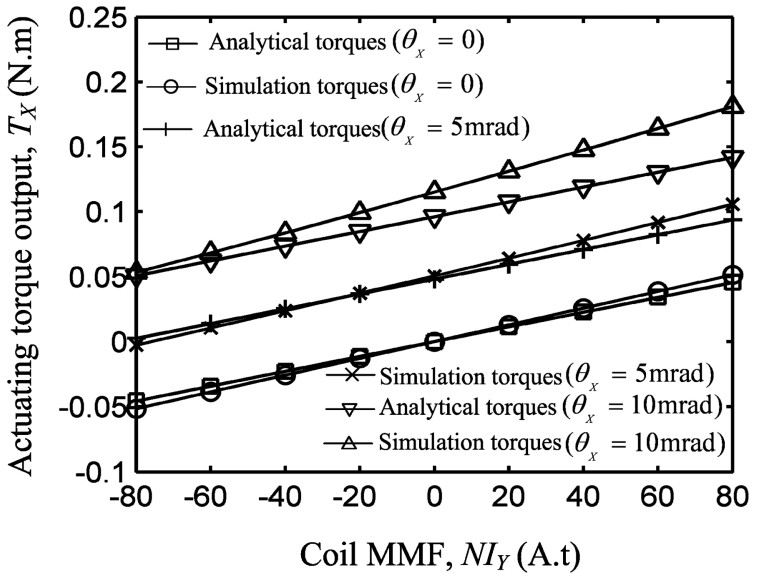

Fig. 15. Variation of torque output with coil MMF.

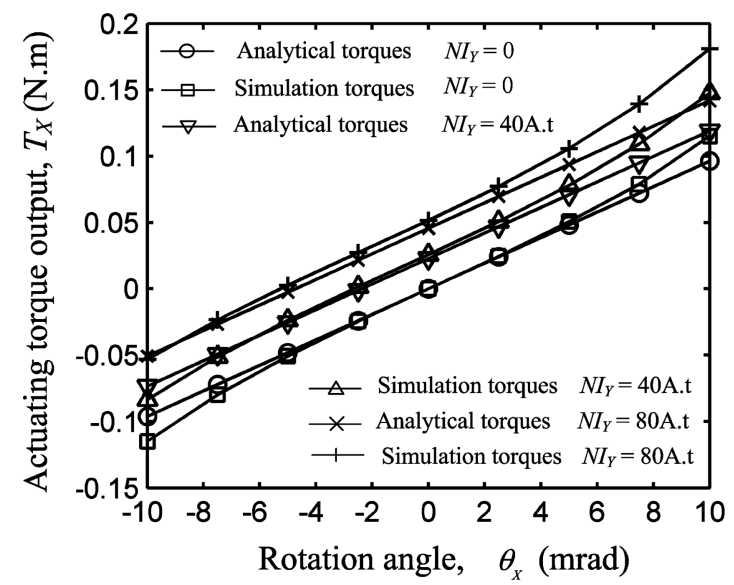

Fig. 16. Variation of torque output with rotation angle.

actuator modeling, the analytical actuator model in this paper is far more accurate than the sAFSM actuator model in Ref. [12].

Figure 16 plots the simulation torque outputs and analytical torque outputs versus the rotation angles. From Fig. 16 , it can be seen that the torque output is nearly proportional to the rotation angle. However, nonlinearity of the simulation torque output is visible and increases with rotation angle. As a result, the relative error of the analytical actuator model becomes greater due to the increase of the rotation angle. Fortunately, the nonlinearity is not obvious in the angular range of $\pm 10 \mathrm{mrad}$ and the analytical actuator model maintains relatively high accuracy in the scanning range of our FSM.

\section{Conclusion}

This paper presents the detailed design of a flux-biased rotary electromagnetic actuator with compact structure for FSM. The actuator works on normal stress and hence has 
high force density. Both the analytical actuator model and finite element analysis results confirm the linear dependence of the actuator's torque output on the excitation current and rotation angle. Benefiting from the new electromagnetic topology, no additional axial force is generated on the cross topology armature which tends to decreases the armature's moment of inertia about the working axis. To improve modeling accuracy, the analytical actuator model is obtained with both the PM flux and coil flux leakages taken into account.

To achieve high dynamic stiffness and long fatigue life, a maximum torque output is preferable. From the perspective of dynamic performance, a maximum torque density is preferable. From the point of view of designing power amplifier, a minimum coil MMF is preferable. But, the three design goals are contradictory. Based on a detailed design, the actuator in this paper achieves a balance between torque output, torque density and required coil MMF. The finite element simulation results validate the design results, along with the concept design, magnetic analysis and torque output model. Our future work will focus on manufacturing a prototype of the actuator, and then demonstrating the design with experiment. Then, the real actuator and the other components of our FSM will be assembled together to test control algorithm and to improve the FSM's performances.

\section{Acknowledgements}

This project is supported by National Natural Science Foundation of China (Grant No. 51375303).

\section{References}

[1] G. C. Loney, Proc. SPIE 1454, 198 (1991).

[2] K. Aoki, Y. Yanagita, H. Kuroda, and K. Shiratama, Proc. SPIE 5160, 495 (2004).

[3] X. Wu, S. Chen, B. Shi, W. Chen, and X. Xiong, Opt. Eng. 50, 023002 (2011).

[4] Y. Lu, D. Fan, and Z. Zhang, Optik 124, 2443 (2013).

[5] S. Woody and S. Smith, Mechatronics 16, 389 (2006).

[6] S. Bing, R. Weibin, G. Bin, R. Changhai, and S. Lining, Smart Mater. Struct. 17, 025032 (2008).

[7] Y. Long, C. Wang, X. Dai, X. Wei, and S. Wang, J. Magn. 19, 130 (2014).

[8] X. Lu, Ph.D. Thesis, Massachusetts Institute of Technology, America (2005).

[9] D. Wu, X. Xie, and S. Zhou, IEEE Trans. Magn. 46, 1007 (2010).

[10] R. C. Montesanti, Ph.D. Thesis, Massachusetts Institute of Technology, America (2005).

[11] D. P. Cuff, M.S. Thesis, Massachusetts Institute of Technology, America (2006).

[12] D. J. Kluk, M. T. Boulet, and D. L. Trumper, Mechatronics 22, 257 (2012). 